\title{
Spin-dependent hybridization between molecule and metal at room temperature through interlayer exchange coupling
}

Manuel Gruber, Fatima Ibrahim, Samy Boukari, Loïc Joly, Victor Da Costa, Michal Studniarek, Moritz Peter, Hironari Isshiki, Hashim Jabbar, Vincent Davesne, Jacek Arabski, Edwige Otero, Fadi Choueikani, Kai Chen, Philippe Ohresser, Wulf Wulfhekel, Fabrice Scheurer, Eric Beaurepaire, Mebarek Alouani, Wolfgang Weber, Martin Bowen

\section{Calibration of the Co and $\mathrm{Cu}$ deposition rates}

We used the variable-temperature scanning tunneling microscope (STM) of the DEIMOS beamline to precisely calibrate our evaporation sources prior to the x-ray absorption measurements. For both elements, we chose substrates such that the given element grows in an island mode so as to allow an easy and precise extraction of the coverage. For Co calibration, we have therefore chosen $\mathrm{Cu}(111)$ and $\mathrm{Au}(111)$ substrates where Co is known to form 2ML-high islands ${ }^{1,2}$. In Fig. S1a, we show a room-temperature topography of Co deposited on $\mathrm{Au}(111)$ held at room temperature. Note that the gold reconstruction is still visible. The height profile along one of the islands is represented in Fig. S1c and confirms that the island is $2 \mathrm{ML} \mathrm{high}^{2}$. From the topography presented in Fig. S1a, the Co coverage can be extracted by taking the ratio of the surface occupied by the islands to the total surface, and multiplying the result by 2 to account for the bilayer growth mode (0.22 ML for the topography presented in Fig. S1a). We repeated the procedure using different topographies taken at different areas of the samples in order to minimize the effect of a possible inhomogeneous deposition of Co. We prepared about 6 different samples with different Co thicknesses and we estimate that the precision of the given Co thicknesses is better than $0.1 \mathrm{ML}$.

For $\mathrm{Cu}$ calibration, we used $\mathrm{Au}(111) / \mathrm{Co}$ and $\mathrm{Au}(111)$ substrates. In Fig. S1b we show a topography of $\mathrm{Cu}$ deposited on $\mathrm{Au}(111)$ held at $190 \mathrm{~K}$. We also show the height profile of one of the islands that indicates a 1ML-high island (see Fig. S1c). The presented results are in agreement with those of Ref. 3. Using the same procedure as for Co, we can extract a coverage of $0.23 \mathrm{ML}$ from the topography shown in Fig. S1b. Considering the coverage determined from different topographies from different samples, we estimate that the precision of the given $\mathrm{Cu}$ thicknesses is better than $0.1 \mathrm{ML}$. Note that, since the very first $\mathrm{Cu}$ atoms incorporate into the $\mathrm{Au}(111)$ substrate $^{3}$, we used $\mathrm{Cu}$ coverage larger than 0.1 ML in order to avoid an important error in the coverage determination.

Note also that the we performed the coverage determination, i.e. the deposition rates determination, on a (111) surface, while our main system of investigation is a $\mathrm{Cu}(001)$ surface. We corrected the deposition rates extracted from the STM measurements to account for the different packing on (111) and (001) surfaces.

As stated in the manuscript, we crafted a $\mathrm{Cu}$ wedge by moving a shutter in front of the sample during the $\mathrm{Cu}$ deposition (with a well-determined deposition rate as described above). We monitored the $\mathrm{Cu}$ wedge by measuring the $\mathrm{x}$-ray absorption edge jump at the $L_{3} \mathrm{Cu}$ edge as a function of the vertical position on the $\mathrm{Cu}(001) / / \mathrm{Co} / \mathrm{Cu} / \mathrm{Co} / \mathrm{MnPc}$ stack 
(this can be similarly done on the $\mathrm{Cu}(001) / / \mathrm{Co} / \mathrm{Cu} / \mathrm{MnPc}$ stack by measuring the x-ray absorption edge jump at the $L_{3}$ Co edge). We could clearly see a constant increase of the $\mathrm{Cu}$ x-ray absorption edge as we moved along the wedge. However, the onset of the wedge was not perfectly visible (most probably due to shadowing effects). We estimated the possible error to be about $0.5 \mathrm{ML}$. In order to properly place the onset of the wedge, we used the well-known interlayer exchange coupling (IEC) oscillations of the prototypical $\mathrm{Cu}(001) / / \mathrm{Co} / \mathrm{Cu} / \mathrm{Co}$ system.

Once the onset of the wedge was precisely fixed and the deposition rate known, any position on the investigated sample can be translated into a $\mathrm{Cu}$ thickness.

Note that the calibration we carried out is very precise but the x-ray beam has a finite size (about $200 \mu \mathrm{m}$ along the wedge direction). In turn, the finite size of the beam causes a convolution over a $\mathrm{Cu}$ thickness of about $0.7 \mathrm{ML}$
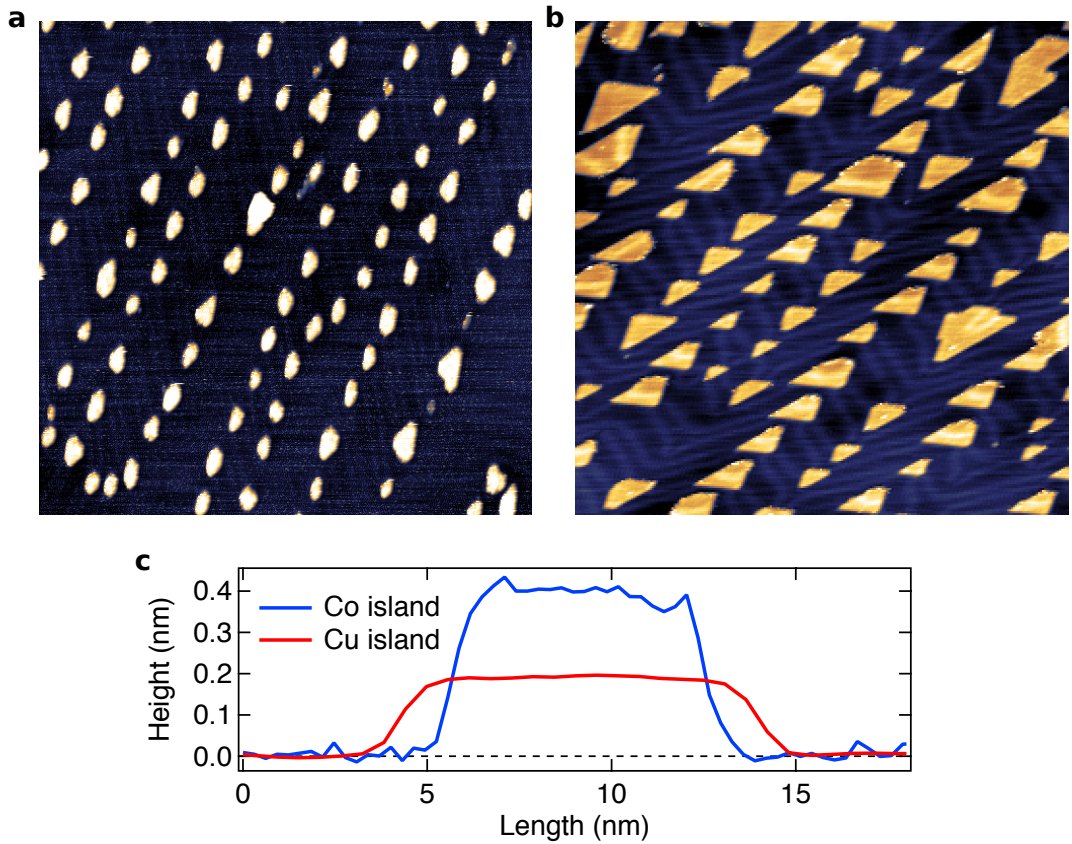

Figure S1: Calibration of the $\mathrm{Co}$ and $\mathrm{Cu}$ sources. Room-temperature STM topographies of a, Co and b, Cu on $\mathrm{Au}(111)$. c, Height profile over Co and $\mathrm{Cu}$ islands. Image sizes are 150x150 nm². 


\section{Growth of MnPc on $\mathrm{Cu}(001)$ and $\mathrm{Cu}(001) / \mathrm{Co}$}

The x-ray absorption results presented in the manuscript indicate a strong interlayer exchange coupling on the $\mathrm{Cu}(001) / / \mathrm{Co} / \mathrm{Cu} / \mathrm{MnPc}$ system. We notably observe a ferromagnetic coupling between the $\mathrm{Mn}$ ion within MnPc and the Co substrate, which evolves to an antiferromagnetic coupling when MnPc and Co are separated by $\sim 1.5 \mathrm{ML}$ of $\mathrm{Cu}$. The conclusion we draw could eventually differ if the growth of MnPc is relatively different on the two surfaces (e.g. if clusters of molecules are formed). We therefore performed low-temperature STM measurements to ensure that the growth at room temperature of MnPc is similar on $\mathrm{Cu}(001)$ and on $\mathrm{Cu}(001) / \mathrm{Co}$.

In Fig. S2a, we show a STM topography of sub-monolayer coverage of MnPc deposited on $\mathrm{Cu}(001)$ held at room temperature. The topography reveals flat-lying $\mathrm{MnPc}$ molecules on the $\mathrm{Cu}(001)$ surface. Moreover, we observe a relatively homogeneous distribution of MnPc over the surface. The molecules remain "isolated" rather than forming molecular clusters.

The distribution of the MnPc molecules on $\mathrm{Cu}(001) / \mathrm{Co}(10 \mathrm{ML})$ is very similar to that found on $\mathrm{Cu}(001)$ as can be observed in Fig. S2b. Note that the $\mathrm{Cu}(001) / C o$ substrate is annealed to $370 \mathrm{~K}$ during $10 \mathrm{~min}$ in order to increase the size of the terraces ${ }^{4,5}$. The molecules are deposited on the substrate held at room temperature.

From the topography shown in Fig. S2b, we also observe that the size of the Co terraces is much larger than that of the MnPc molecules. Considering the growth of the MnPc molecules and the lateral size of the Co terraces, we can expect the MnPc molecules to be insensitive to the Co roughness. Note that the topography presented in Fig. S2b gives insight into the roughness of the first Co layer: we can observe about 3 atomic layers. The dominant Co layer covers about $60 \%$ of the surface $(26 \%$ and $13 \%$ for the upper and lower Co layers, respectively).

We didn't measure MnPc deposited on $\mathrm{Cu}(001) / \mathrm{Co} / \mathrm{Cu}$. However, STM studies have been carried out on such surfaces and shows that the terraces can be much larger than the molecular size provided that the last $\mathrm{Cu}$ layer is deposited at low temperature ${ }^{6}$. We have therefore strong reasons to believe that the growth of $\mathrm{MnPc}$ on $\mathrm{Cu}(001) / \mathrm{Co} / \mathrm{Cu}$ is similar to that on $\mathrm{Cu}(001)$.

Finally, we mention that we calibrated our MnPc source by measuring different coverage of $\mathrm{MnPc}$ on $\mathrm{Cu}(001)$ such as shown in Fig. S2a, using the room-temperature STM of the DEIMOS beamline.
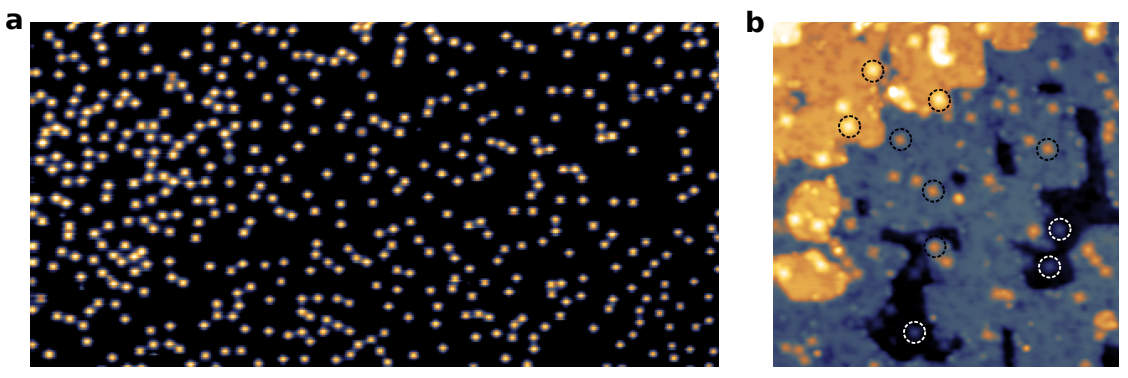

Figure S2: Growth of $\mathrm{MnPc}$ on $\mathrm{Cu}(001)$ and $\mathrm{Cu}(001) / C o$. Low-temperature STM topographies of submonolayer coverage of $\mathrm{MnPc}$ on $\mathbf{a}, \mathrm{Cu}(001)$ and $\mathbf{b}, \mathrm{Cu}(001) / \mathrm{Co}$. Single molecules are encircled in $\mathbf{b}$ for clarity. Image sizes are $\mathbf{a}, 80 \times 40 \mathrm{~nm}^{2}$ and $\mathbf{b}, 40 \times 40 \mathrm{~nm}^{2}$. 


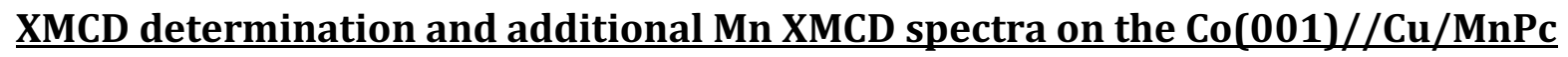
$\underline{\text { stack }}$

In order to obtain one XMCD spectra we acquired 8 different XAS spectra: $\mu^{++}, \mu^{+}, \mu^{++}, \mu^{++}$, $\mu^{-+}, \mu^{--}, \mu^{--}$and $\mu^{-+}$, where the first symbol of the exponent indicates the direction of the external magnetic field and the second symbol represents the helicity of the photons. The definition of the helicity is chosen such that the XMCD of the $L_{3}$ edge of a thin-film ferromagnetic Co layer is negative ${ }^{7}$. We then take the average of the equivalent quantities: $\mu_{+}=\left(\mu^{++}+\mu^{++}+\mu^{-+}+\mu^{-}\right) / 4$ and $\mu_{-}=\left(\mu^{++}+\mu^{++}+\mu^{++}+\mu^{-+}\right) / 4$. The XMCD spectra are obtained by taking the difference: $\mu_{+}-\mu$. In Fig. $2 \mathrm{c}$ of the manuscript, we also represent averaged XAS spectra, which were obtained by taking the average: $\left(\mu_{+}+\mu_{-}\right) / 2$.

We also would like to stress that the same arbitrary units are used in all the XAS and XMCD spectra at the Mn edges and are therefore comparable.

In Fig. S3, we show Mn XMCD spectra acquired on the Co(001)/Cu/MnPc stack at $4.8 \mathrm{~K}$ in an external magnetic field of $0.1 \mathrm{~T}$ for different $\mathrm{Cu}$-spacer thicknesses $d_{c u}$ ranging from 1.5 to $12.8 \mathrm{ML}$. For $d_{C u}=1.5 \mathrm{ML}$, the $L_{3}$ edge of the XMCD is positive while that of Co is negative (not shown), implying an antiferromagnetic (AF) alignment of the Mn within MnPc and Co moments. This is in line with the similar Mn XMCD spectrum measured at room temperature (see Fig. $2 \mathrm{~d}$ of the manuscript). For increasing $\mathrm{Cu}$ thicknesses, the sign of the XMCD at the $L_{3}$ edge oscillates from negative to positive (see Fig. S3). This implies that the magnetic coupling between the Mn within MnPc and Co moments oscillates from ferromagnetic (FM) to AF with increasing Cu thicknesses. As discussed in the manuscript, this oscillation arises from the oscillatory interlayer exchange coupling that stabilizes the moment of the Mn ions within MnPc. Moreover, Fig. S3 shows that this exchange interaction is still not negligible with a $\mathrm{Cu}$ spacer as large as 12.8 ML.

As a secondary effect compared to the primary effect of the XMCD sign change, we observe that the XMCD line shape and peak position evolve with $d_{C u}$. This effect, which is beyond the scope of this work, is expected. Indeed, for certain thicknesses, quantum well states provide a spin-polarized modulation of the DOS, notably at and around the Fermi level $\mathrm{E}_{\mathrm{F}}{ }^{8}$. The spin-polarized hybridization with $\mathrm{Mn}$ is therefore evolving with $d_{C u}$ (see the calculated partial density of states of the Mn ion in Fig. S5). This can naturally alter the line shape and peak position of XMCD. Such changes to XMCD spectra have for instance been observed at the $\mathrm{Cu} \mathrm{K}$ edge upon varying $d_{C u}$ in $\left[\mathrm{Co} / \mathrm{Cu}\left(d_{C u}\right)\right]_{\mathrm{n}}$ multilayers ${ }^{9}$, with changes in peak position reaching $\sim 2 \mathrm{eV}$. 


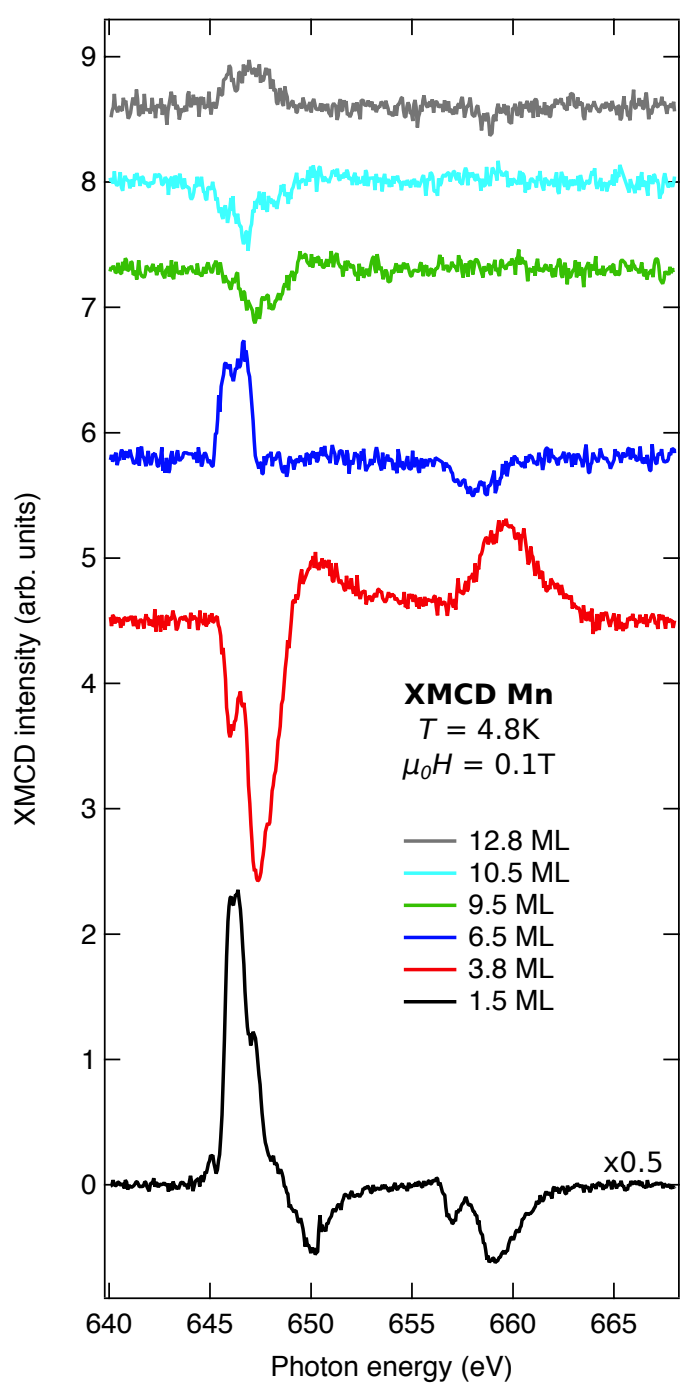

Figure S3: XMCD spectra acquired at the Mn $L$ edges at $4.8 \mathrm{~K}$ in an external magnetic field of $0.1 \mathrm{~T}$ on $\mathrm{Co}(001) / \mathrm{Cu}(\mathrm{x} \mathrm{ML}) / \mathrm{MnPc}$ where $\mathrm{x}$ ranges from 1.5 to $12.8 \mathrm{ML}$. The sign of the XMCD $L_{3}$ edge oscillates with increasing $\mathrm{Cu}$-spacer thickness. Note that the XMCD intensity for $1.5 \mathrm{ML}$ of $\mathrm{Cu}$ is divided by two. 


\section{Experimental estimation of the interlayer exchange coupling strength}

The paramagnetic manganese ion of MnPc molecules has a total spin $S=3 / 2$. When an external magnetic field $H$ is applied, a proportion of the Mn magnetic moments tends to align towards the external field, and the resulting normalized magnetization is given by the Brillouin function:

$$
B_{J}\left(\frac{\mu_{0} H}{T}\right)=\frac{2 J+1}{2 J} \operatorname{coth}\left(\frac{2 J+1}{2 J} \frac{g \mu_{B} J}{k_{B}} \frac{\mu_{0} H}{T}\right)-\frac{1}{2 J} \operatorname{coth}\left(\frac{1}{2 J} \frac{g \mu_{B} J}{k_{B}} \frac{\mu_{0} H}{T}\right)
$$

with $T$ the temperature, $J$ the total angular momentum quantum number that we will consider equal to $S=3 / 2, g$ the Landé factor taken equal to $2, \mu_{B}$ the Bohr magneton and $k_{B}$ the Boltzmann constant.

On a $\mathrm{Cu}(001) / / \mathrm{Co} / \mathrm{Cu} / \mathrm{MnPc}$ sample, we acquired XMCD spectra at the $\mathrm{Mn} L$ edges for different temperatures and applied magnetic fields for $\mathrm{Cu}$ thicknesses of 1.5 and $7 \mathrm{ML}$ (see Fig. S4a). We observe that, for a low applied magnetic field of $0.1 \mathrm{~T}$, the corresponding XMCD intensities have non-zero values, while the sign of XMCD implies that the direction of the Mn magnetic moments is opposed to that of the applied magnetic field. This can be understood if we take into account that the Mn magnetic moments do not only feel the applied magnetic field $H$ but also the exchange field $H_{e x}$ which comes from the indirect interlayer exchange coupling across $\mathrm{Cu}$. Thus, the $\mathrm{Mn}$ magnetic moments feel an effective magnetic field $H_{e f f}=H+H_{e x}$. If $H_{e x}$ is now both opposed to $H$ and stronger in amplitude, the effective field $H_{\text {eff }}$ is opposed to $\mathrm{H}$ as well and leads thus to an antiparallel alignment of the Mn magnetic moments with respect to the Co reference magnetization.

One can extract the exchange magnetic fields $H_{e x}^{1.5 M L}$ and $H_{e x}^{7 M L}$ for 1.5 and $7 \mathrm{ML} \mathrm{Cu}$, respectively, by fitting the XMCD intensities as a function of temperature and external applied magnetic field with a Brillouin function in which the external applied field $H$ is replaced by the effective field $H_{e f f}$. We find a strong exchange field of $\mu_{0} H_{e x}^{1.5}=-78( \pm 38) \mathrm{T}$ for 1.5 ML of $\mathrm{Cu}$. As expected, the interlayer exchange coupling strength, and thus the exchange field is reduced for $7 \mathrm{ML}$, for which we find $\mu_{0} H_{e x}^{7}=-0.8( \pm 0.5) \mathrm{T}$. The relatively high uncertainty in the exchange field amplitude arises only from the limited number of points, while this approach to measuring the interlayer exchange coupling strength, expressed in magnetic field units, is in principle accurate. 

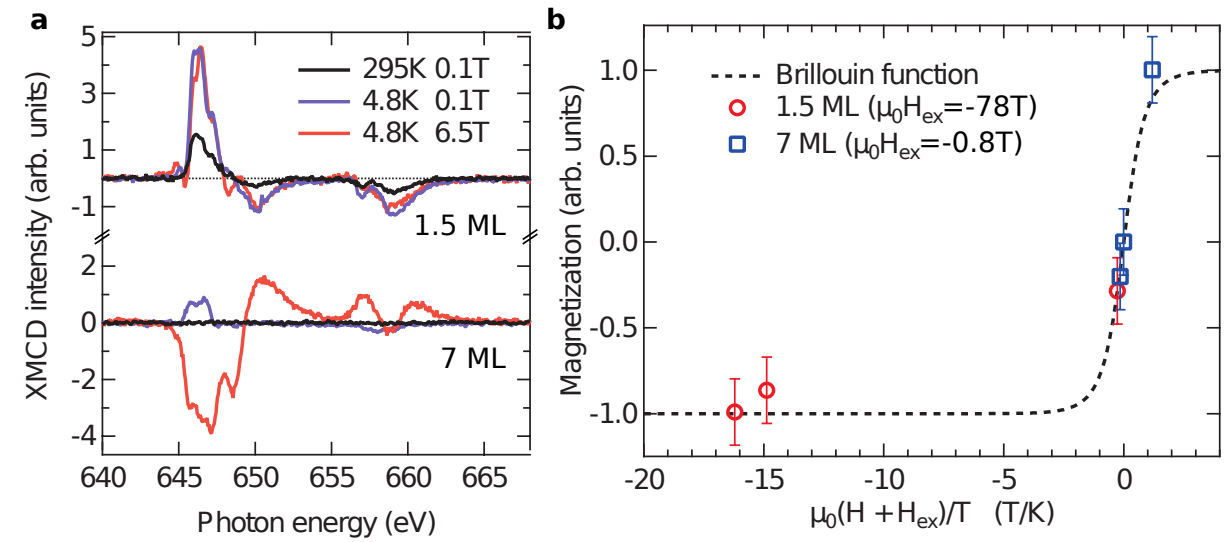

Figure S4: Estimation of the interlayer exchange coupling strength. a, XMCD spectra at Mn $L$ edges acquired for different temperatures and applied magnetic fields on $\mathrm{Co}(001) / \mathrm{Cu} / \mathrm{MnPc}$ for $\mathrm{Cu}$ thicknesses of 1.5 and $7 \mathrm{ML}$. For 1.5 ML of $\mathrm{Cu}$ (upper panel), the XMCD spectra indicate an anti-alignment of the Mn moment with the applied magnetic field, suggesting an exchange field higher than $6.5 \mathrm{~T}$. For $7 \mathrm{ML}$ of $\mathrm{Cu}$ (lower panel), there is no visible dichroism at room temperature, and at low temperature, the XMCD is reversed when going from $0.1 \mathrm{~T}$ to $6.5 \mathrm{~T}$, indicating an exchange field between $0.1 \mathrm{~T}$ and $6.5 \mathrm{~T}$. b, Results of the fit of the XMCD intensities as a function of temperature and applied magnetic field with a Brillouin function in which the external applied magnetic field $H$ is replaced by the effective field $H_{e f f}=H+H_{e x}$. The free parameters of the fit are the exchange magnetic fields for Cu thicknesses of 1.5 and $7 \mathrm{ML}$. 


\section{$\underline{A b \text { initio calculations of magnetic and spintronic properties of the } \mathrm{Co} / \mathrm{Cu} / \mathrm{MnPc} \text { stack }}$}

We have conducted $a b$ initio calculations to investigate the magnetic interaction between MnPc molecule and $\mathrm{Co}(001)$ surface separated by $\mathrm{n}(\mathrm{n}=0, \ldots, 3) \mathrm{Cu}(001)$ layers. To do so, we used the VASP package ${ }^{10}$ and the generalized gradient approximation for exchange-correlation potential as parametrized by Perdew, Burke, and Ernzerhof ${ }^{11}$. We used the projector augmented wave (PAW) pseudopotentials as provided by VASP ${ }^{12}$. FcC $\mathrm{Co}(001)$ and $\mathrm{Cu}(001)$ surfaces were modeled by using a supercell of 3 atomic monolayers of $8 \times 8$ atoms separated by a vacuum region. Since experiments used cobalt epitaxially grown on $\mathrm{Cu}$, we used the $f c c$ lattice parameter of $0.36 \mathrm{~nm}$ for both cobalt and copper. A kinetic energy cutoff of $450 \mathrm{eV}$ was used for the plane-wave basis set and the gamma point to sample the first Brillouin zone. We used the optimized structure of the MnPc molecule adsorbed at $2.1 \AA$ away from fcc Co(001) surface as in Ref. 13. Furthermore, the optimized structure of the MnPc molecule adsorbed at $2.36 \AA$ away from the $\mathrm{Cu}$ surface was reproduced by including the van-der-Waals interactions within GGA-D2 approach ${ }^{14,15}$ as detailed in Ref. 16.

\begin{tabular}{|c|c|c|c|c|}
\hline & $\boldsymbol{d}_{\mathrm{Cu}}=\mathbf{0}^{13}$ & $d_{\mathrm{Cu}}=1 \mathrm{ML}$ & $d_{\mathrm{Cu}}=2 \mathrm{ML}$ & $d_{\mathrm{Cu}}=3 \mathrm{ML}$ \\
\hline \multicolumn{5}{|l|}{ Magnetic moments $\left(\mu_{B}\right)$} \\
\hline $\mathrm{Mn}$ & +2.54 & -2.78 & +2.79 & +2.8 \\
\hline $\mathrm{N}_{n n}$ & +0.01 & +0.1 & -0.1 & -0.1 \\
\hline $\mathrm{N}_{f n}$ & +0.1 & -0.04 & +0.05 & +0.01 \\
\hline $\mathrm{C}$ & -0.22 & +0.08 & -0.18 & -0.21 \\
\hline \multicolumn{5}{|l|}{ Total Energies } \\
\hline $\mathrm{E}_{\mathrm{FM}}(\mathrm{eV})$ & -1657.622 & -1899.199 & -2135.048 & -2374.552 \\
\hline $\mathrm{E}_{\mathrm{AF}}(\mathrm{eV})$ & -1657.184 & -1899.315 & -2135.013 & -2374.549 \\
\hline$\Delta \mathrm{E}(\mathrm{eV})$ & -0.438 & +0.116 & -0.035 & -0.003 \\
\hline$\mu_{0} \mathrm{H}_{\mathrm{ex}}(\mathrm{T})$ & +1490 & -360 & +110 & +12 \\
\hline \multicolumn{5}{|l|}{ Densities of States } \\
\hline z-DOS (arb. units) & 0.25456 & 0.04283 & 0.15732 & 0.20961 \\
\hline pl-DOS (arb. units) & 0.03322 & 0.0044 & 0.0096 & 0.00675 \\
\hline z-DOS/Pl-DOS & 7.66 & 9.72 & 16.39 & 31.05 \\
\hline $\mathrm{z}-\mathrm{P}\left(\mathrm{E}_{\mathrm{F}} \pm 25 \mathrm{meV}\right)(\%)$ & 65.8 & -98.3 & 30 & 74 \\
\hline $\mathrm{pl}-\mathrm{P}\left(\mathrm{E}_{\mathrm{F}} \pm 25 \mathrm{meV}\right)(\%)$ & -69 & -99.2 & 17 & 51.1 \\
\hline Total P $\left(\mathrm{E}_{\mathrm{F}} \pm 25 \mathrm{meV}\right)(\%)$ & 50.2 & -98.4 & 29.3 & 73.3 \\
\hline
\end{tabular}

TABLE I. Computational results of the $f c c \operatorname{Co}(001) / C u\left(d_{\mathrm{Cu}}\right) / \mathbf{M n P c}$ stack. Calculated spin on the atomic sites of MnPc as a function of the number of Cu MLs inserted between MnPc and Co(001). We denote by $\mathrm{N}_{n n}$ the nitrogen atoms that are nearest neighbors to $\mathrm{Mn}$ and $\mathrm{N}_{f n}$ those that are far. The magnetic moment of Co is positive, while the spin polarization of Co at $E_{F}$ is negative.

Table I summarizes our findings on the theoretically determined magnetic coupling between MnPc molecules and Co across $\mathrm{Cu}$. The strong chemisorption of the MnPc molecule to the Co surface and ensuing FM coupling is reflected through the large $0.44 \mathrm{eV}$ exchange coupling. As mentioned in the main text, inserting $\mathrm{Cu}$ monolayers (ML) between $\mathrm{Co}$ and MnPc leads to an oscillation in sign of the magnetic coupling strength. Indeed, AF coupling is strongly favored for $1 \mathrm{ML} \mathrm{Cu}$, while weaker FM coupling is predicted for $2 \mathrm{ML}$ and $3 \mathrm{ML} \mathrm{Cu}$. 
We may relate the difference in energy $\Delta E=E_{A F}-E_{F M}$ to the exchange field $H_{e x}$ as: ${ }^{\boldsymbol{\mu}_{0} H_{\theta x}}=\frac{-\Delta E}{2 \mu_{M o l}}$, where $\mu_{\text {Mol }}$ is the molecule's average magnetic moment. As summarized in Table I, $H_{e x}$ strongly decreases when $\mathrm{Cu}$ is inserted, as direct exchange is replaced by interlayer exchange. We nevertheless find enduring values of $12 \mathrm{~T}$ for $3 \mathrm{ML}$ $\mathrm{Cu}$, which are thus in qualitative agreement with experiment (see previous Note).

The magnetic moment on the Mn site jumps from $2.54 \mu_{\mathrm{B}}$ to $2.78 \mu_{\mathrm{B}}$ for $1 \mathrm{ML} \mathrm{Cu}$ as the spin-polarized charge transfer decreases when going from a $\mathrm{Co}$ to a $\mathrm{Cu}$ surface (see results of a Bader analysis shown in Table II). With increasing $\mathrm{Cu}$ thickness, the $\mathrm{Mn}$ magnetic moment trends upwards toward that found in a free molecule $\left(3.1 \mu_{\mathrm{B}}\right)$. Overall, the magnetic moment on the $\mathrm{N}$ and $\mathrm{C}$ sites remains mostly unchanged upon $\mathrm{Cu}$ insertion.

In Fig. 2c of the manuscript, the peak shape of the XAS at the Mn $L_{3}$ edge evolves with the underlying $\mathrm{Cu}$ thickness. This evolution of the peak shape suggests a different orbital alignment, ligand field and/or a different charge state of the central $\mathrm{Mn}$ ion. In order to check this point, we estimated the charge of the different elements composing the MnPc molecule from our calculations by performing a Bader analysis. We present the results in Table II. Note that the Bader charge analysis yields qualitative results and usually underestimates the charges compared to other charge-analysis methods. We can see that the MnPc molecule experiences a charge transfer when it is deposited on $f c c$ $\mathrm{Co}(001)$ or on $f c c \mathrm{Co}(001) / \mathrm{Cu}$. Furthermore, the charge transfer is more important when the molecule lies on $f c c \mathrm{Co}(001)(-3.5|\mathrm{e}|$ relative to $\sim-1.4|\mathrm{e}|$ when the MnPc molecule lies on $f c c \mathrm{Co}(001) / \mathrm{Cu})$.

In addition, we observe that the element-resolved Bader charges vary with the substrate and notably with the number of underlying $\mathrm{Cu}$ layers $\mathrm{n}$.

\begin{tabular}{|l|l|l|l|l|c|}
\hline $\begin{array}{l}\text { Bader } \\
\text { charge }\end{array}$ & $\mathbf{n = 0}$ & $\mathbf{n = 1}$ & $\mathbf{n = 2}$ & $\mathbf{n = 3}$ & $\begin{array}{c}\text { Free } \\
\text { molecule }\end{array}$ \\
\hline $\begin{array}{l}\text { MnPc } \\
\text { (total) }\end{array}$ & -3.5 & -1.5 & -1.40 & -1.39 & 0 \\
\hline $\mathrm{Mn}$ & +1.03 & +1.101 & +1.112 & +1.113 & +1.246 \\
\hline $\mathrm{N}$ & -9.4 & -9.739 & -9.756 & -9.761 & -9.546 \\
\hline $\mathrm{C}$ & +3.04 & +5.95 & +6.007 & +6.011 & +7.363 \\
\hline
\end{tabular}

TABLE II. Results of the Bader analysis of a free MnPc molecule and MnPc on the fcc $\operatorname{Co}(3 \mathrm{ML}) / \mathrm{Cu}\left(\boldsymbol{d}_{\mathrm{Cu}}\right)$ stack. All the charges are expressed in unit of the elementary charge $|\mathrm{e}|$.

The Bader analysis can reasonably well explain the different $\mathrm{Mn} L_{3}$ edge peak shape of the XAS/XMCD for 0 and 1 ML of intercalated $\mathrm{Cu}$. However, the Mn Bader charges for $\mathrm{n}=$ 1, 2 and 3 are very similar and thus cannot explain the observed shift of the Mn $L_{3}$ edge of about $1 \mathrm{eV}$. One should therefore have a closer look at the partial density of states (PDOS) of the Mn ion within MnPc on $f c c \mathrm{Co}(001) / \mathrm{Cu}(\mathrm{n} \mathrm{ML})$ shown in Fig. S5. The calculated Mn PDOS clearly reveals large unoccupied DOS at three main energies 
separated by about $1 \mathrm{eV}$ (see the PDOS represented in blue in Fig. S5). The shape and the energy distribution of the unoccupied DOS change with the number of $\mathrm{Cu}$-spacer layers n. In turn, the XAS/XMCD line-shape mainly depends on the energetic positions of the unoccupied orbitals, transition probabilities and electron-hole interaction.

The observed change in the XAS/XMCD line shape with increasing $\mathrm{Cu}$ spacer thickness may thus reflect a changing spectral weight of the three groups of unoccupied states (Fig. S5).

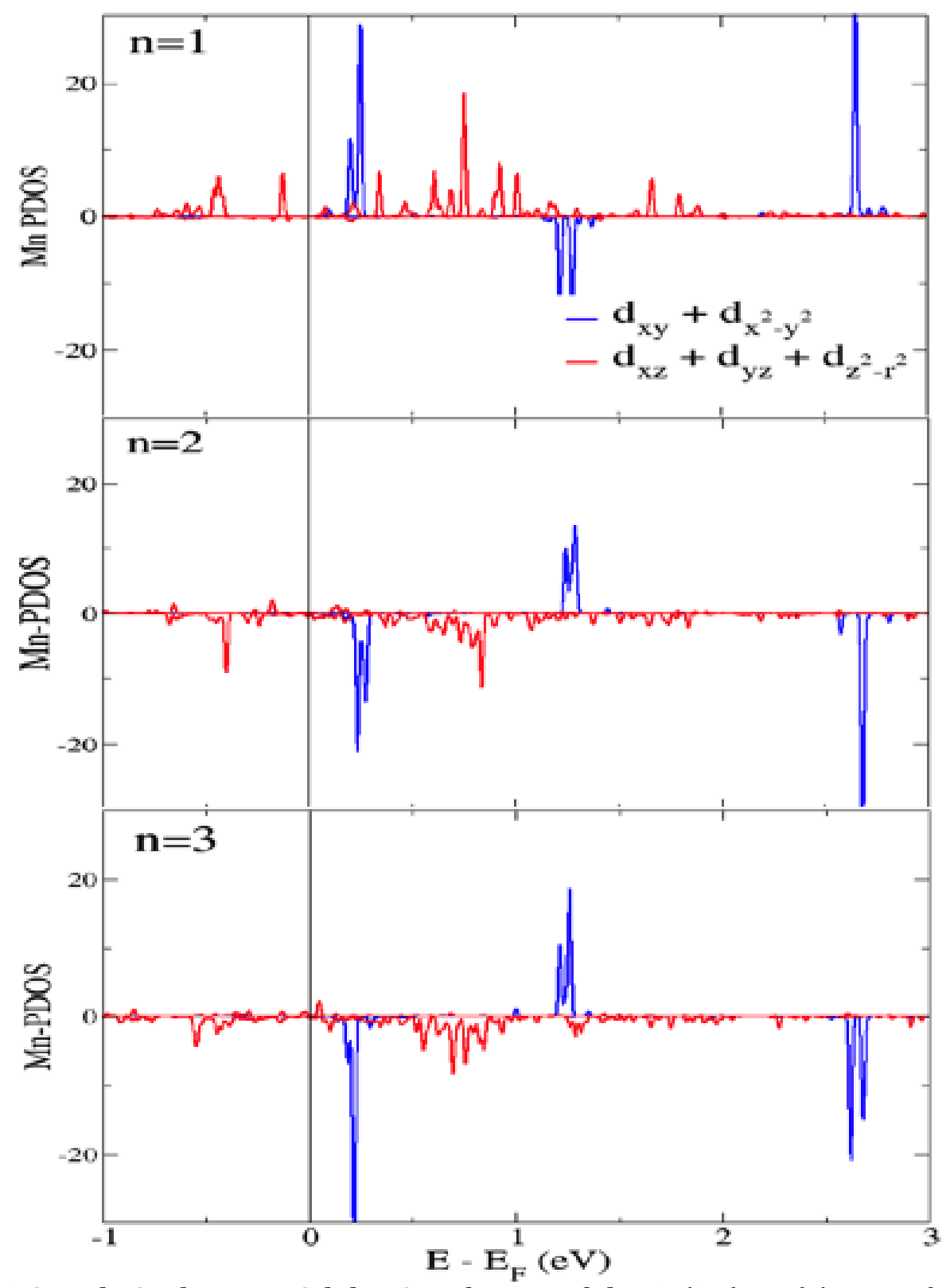

Figure S5: Spin-polarized Mn partial density of states of the Co/Cu(n ML)/MnPc. The PDOS arising from the $d_{x y}$ and $d_{x}{ }^{2}-y^{2}\left(d_{x z}, d_{y z}\right.$ and $\left.d_{z}^{2}-r^{2}\right)$ is represented in blue (red). Note that a positive (negative) DOS corresponds to spin-up (spin-down) states. 


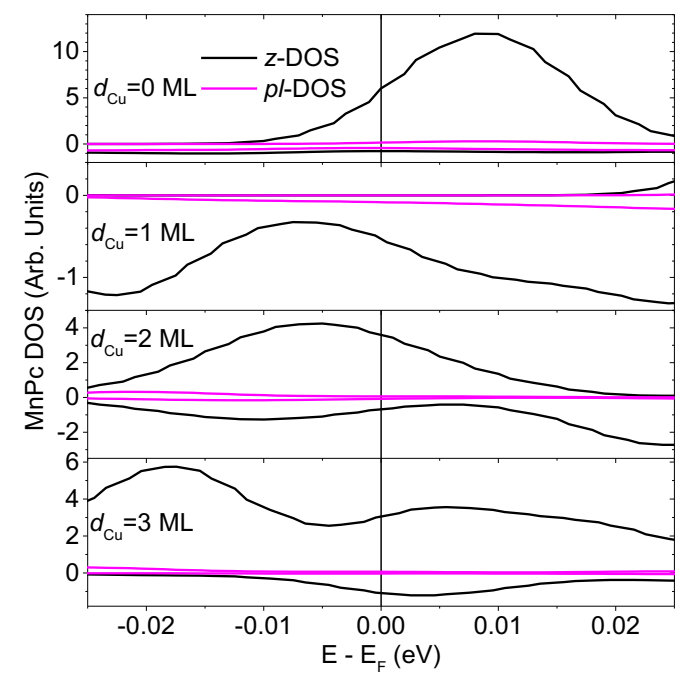

Figure S6: Spin-polarized density of states of the Co/Cu/MnPc stack for several $\mathrm{Cu}$ thicknesses. $z$ DOS within $25 \mathrm{meV}$ of $E_{F}$, which includes only those states which are involved in transport across the metal-molecule interface at room temperature. Planar DOS (pl-DOS) is also represented. Co/Cu/MnPc systems are endowed with spin polarizations that can reach $-98 \%$. Data for $d_{C u}=0$ taken from Ref. ${ }^{13}$. All DOSs were convoluted with a Gaussian distribution of width $0.01 \mathrm{eV}$. Note that a positive (negative) DOS corresponds to spin-up (spin-down) states.

At room temperature, transport will proceed within a $25 \mathrm{meV}$ energy window defined by thermal agitation. We present in panel Fig. S6 the molecule's spin-polarized DOS within this energy window. For bare Co, we find the surface-induced interfacial states described previously ${ }^{13}$. Inserting $1 \mathrm{ML}$ of $\mathrm{Cu}$ strongly decreases the DOS located at the metal-molecule interface. Indeed, nearly no spin $\uparrow$ MnPc z-DOS is present. The spin $\downarrow$ channel is dominated here by C DOS. This leads to $-98 \%$ spin polarization within $\pm 25 \mathrm{meV}$. For $2 \mathrm{ML} \mathrm{Cu}$, we find only $+30 \%$ spin polarization. Finally, for $3 \mathrm{ML} \mathrm{Cu}, \mathrm{C}$ sites contribute a large peak at $E_{F}$, and a small peak slightly above $E_{F}$, leading to $+75 \%$ spin polarization. We note that, in all cases, the bulk of the MnPc z-DOS is located on C sites. Furthermore, we find that inserting $\mathrm{Cu}$ between Co and MnPc strongly attenuates the molecule's planar DOS for $E_{F}-25<E(\mathrm{meV})<E_{F}+25$ (see Fig. S3 and Table 1). We summarize in Table 1 the spintronic properties of the z-DOS and pl-DOS. 

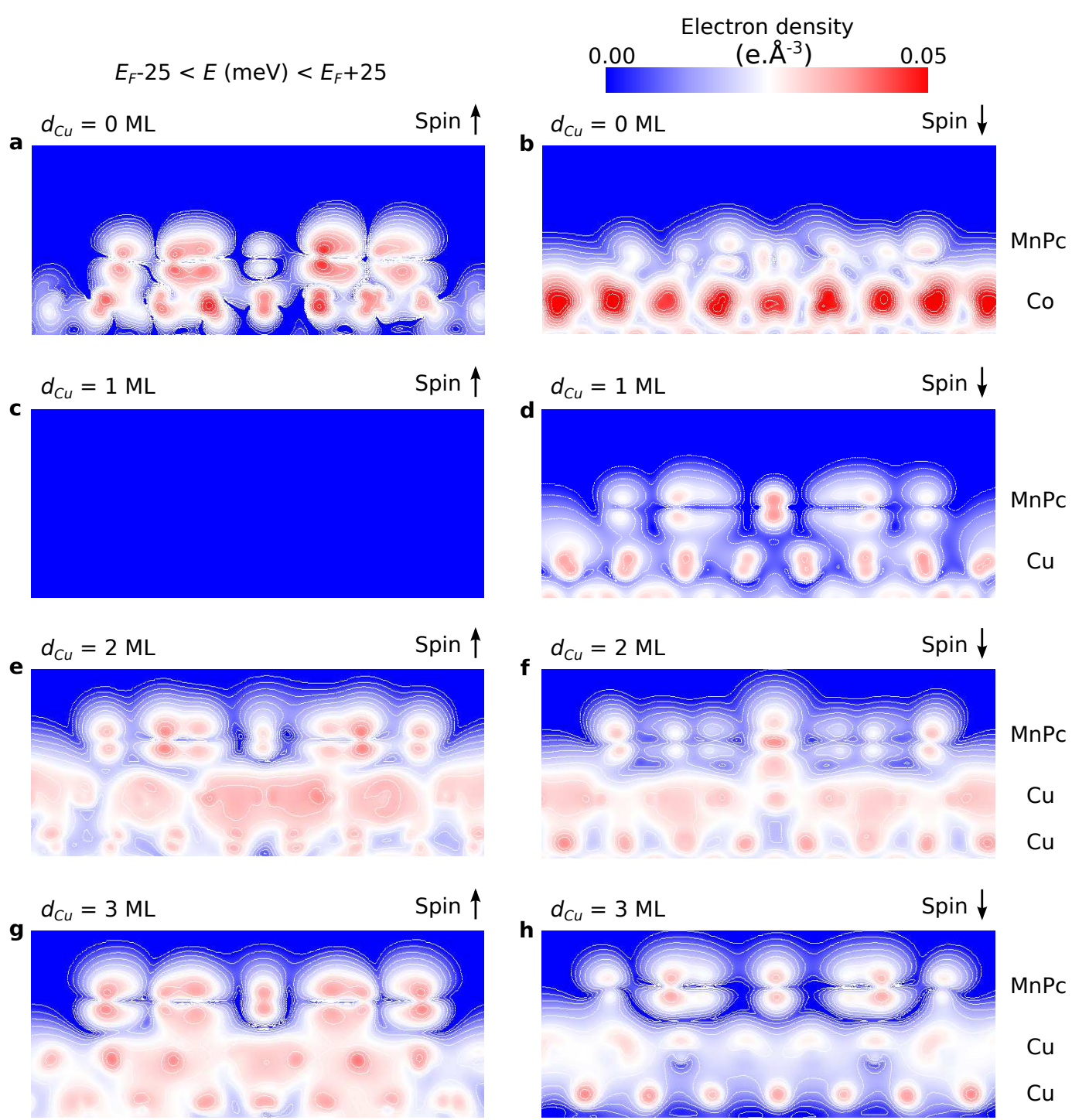

Figure S7: Spin-polarized spatial maps of the Co/Cu/MnPc system for several Cu thicknesses. Spin $\uparrow$ and spin $\downarrow$ maps within $25 \mathrm{meV}$ of $E_{F}$ for $d_{\mathrm{Cu}}=0 \mathrm{ML}$ (the data from panels a and b are published in Ref. 13), $d_{\mathrm{Cu}}=1 \mathrm{ML}$ (panels c and d), $d_{\mathrm{Cu}}=2 \mathrm{ML}$ (panels e and f), and $d_{\mathrm{Cu}}=3 \mathrm{ML}$ (panels $\mathrm{g}$ and $\mathrm{h}$ ). The maps are taken along the dotted line represented in Fig. 4. All maps are plotted with respect to the same magnetization referential of Co. No Gaussian smearing was applied here. Note that for $d_{\mathrm{Cu}}=1 \mathrm{ML}$ (c) there is no spin up density of states in this energy range.

This spin-polarized DOS within $25 \mathrm{meV}$ of $E_{\mathrm{F}}$ will in turn contribute to spin-polarized transport across the interface as weighed by the molecule's hybridization to the underlying $\mathrm{Cu}$ surface. To evaluate this aspect, we present in Fig. S7 spin-polarized spatial maps for all $d_{\mathrm{Cu}}$ considered. One sees that the DOS of the $\mathrm{Cu}$ surface evolves with $d_{C u}$. This reflects both the diminishing impact of Co hybridization with increasing $d_{C u}$, and the impact of the $\mathrm{Cu}$ QWSs. This evolving $\mathrm{Cu}$ interfacial DOS density in turn modulates the hybridization between metal surface and molecule, and the ensuing spintronic properties of the interface. Indeed, while the hybridization is weighted rather evenly in both spin channels for $d_{C u}=2 \mathrm{ML}$, a strong asymmetry for $d_{C u}=3$ ML shall promote a transport spin polarization that is higher than that inferred from DOS considerations alone (see Fig. S3). 


\section{References}

(1) de la Figuera, J.; Prieto, J. E.; Ocal, C.; Miranda, R. Phys. Rev. B 1993, 47 (19), 13043-13046.

(2) Voigtländer, B.; Meyer, G.; Amer, N. M. Phys. Rev. B 1991, 44 (18), 10354-10357.

(3) Grillo, F.; Früchtl, H.; Francis, S. M.; Richardson, N. V. New J. Phys. 2011, 13 (1), 013044 .

(4) Ferrer, S.; Vlieg, E.; Robinson, I. K. Surf. Sci. Lett. 1991, 250 (1-3), L363-L367.

(5) Schmid, A. K.; Atlan, D.; Itoh, H.; Heinrich, B.; Ichinokawa, T.; Kirschner, J. Phys. Rev. B 1993, 48 (4), 2855-2858.

(6) Würsch, C.; Stamm, C.; Egger, S.; Pescia, D.; Baltensperger, W.; Helman, J. S. Nature 1997, 389 (6654), 937-939.

(7) Stöhr, J.; Siegmann, H. C. Magnetism From Fundamentals to Nanoscale Dynamics; Springer-Verlag Berlin Heidelberg: [New York], 2006.

(8) Garrison, K.; Chang, Y.; Johnson, P. D. Phys. Rev. Lett. 1993, 71 (17), 2801-2804.

(9) Pizzini, S.; Fontaine, A.; Baudelet, F.; Minr, S.; Giorgetti, C.; Dartyge, E.; Bobo, J. F.; Piecuch, M. Nucl. Instrum. Methods Phys. Res. Sect. B Beam Interact. Mater. At. 1995, 97 (1-4), 436-443.

(10) Kresse, G.; Furthmüller, J. Phys. Rev. B 1996, 54 (16), 11169-11186.

(11) Perdew, J. P.; Burke, K.; Ernzerhof, M. Phys. Rev. Lett. 1996, 77 (18), 3865-3868.

(12) Kresse, G.; Joubert, D. Phys. Rev. B 1999, 59 (3), 1758-1775.

(13) Djeghloul, F.; Ibrahim, F.; Cantoni, M.; Bowen, M.; Joly, L.; Boukari, S.; Ohresser, P.; Bertran, F.; Le Fèvre, P.; Thakur, P.; Scheurer, F.; Miyamachi, T.; Mattana, R.; Seneor, P.; Jaafar, A.; Rinaldi, C.; Javaid, S.; Arabski, J.; Kappler, J.-P.; Wulfhekel, W.; Brookes, N. B.; Bertacco, R.; Taleb-Ibrahimi, A.; Alouani, M.; Beaurepaire, E.; Weber, W. Sci. Rep. 2013, 3, 1272.

(14) Bučko, T.; Hafner, J.; Lebègue, S.; Ángyán, J. G. J. Phys. Chem. A 2010, 114 (43), 11814-11824.

(15) Grimme, S. J. Comput. Chem. 2006, 27 (15), 1787-1799.

(16) Javaid, S.; Lebègue, S.; Detlefs, B.; Ibrahim, F.; Djeghloul, F.; Bowen, M.; Boukari, S.; Miyamachi, T.; Arabski, J.; Spor, D.; Zegenhagen, J.; Wulfhekel, W.; Weber, W.;

Beaurepaire, E.; Alouani, M. Phys. Rev. B 2013, 87 (15), 155418. 